\title{
Caracterização do perfil epidemiológico de vítimas carbonizadas necropsiadas no IML-SP entre 2010 e 2015
}

\section{Characterization of the epidemiological profile of carbonized victims necropsied in IML-SP between 2010 and 2015}

\author{
Letycia Paiva Andrade ${ }^{1}$, Paulo Sérgio Tieppo Alves ${ }^{2}$, \\ Luiz Roberto Fontes ${ }^{3}$, Ana Cristina Mielli ${ }^{4}$
}

DOI: http://dx.doi.org/10.11606/issn.2317-2770.v22i1p3-8

\begin{abstract}
Andrade LP, Alves PST, Fontes LR, Mielli AC. Caracterização do perfil epidemiológico de vítimas carbonizadas necropsiadas no IML-SP entre 2010 e 2015. Saúde, Ética \& Justiça. 2017;22(1):3-8.

RESUMO: O monóxido de carbono faz parte do grupo dos poluentes gasosos e pode agir como um poderoso asfixiante químico, sendo responsável por elevadas taxas de mortalidade no mundo. Quando inalada, essa substância se difunde facilmente pela membrana alvéolo-capilar, e, uma vez na corrente sanguínea, liga-se facilmente à hemoglobina, devido à alta afinidade existente entre esta molécula e o monóxido de carbono. Consequentemente, quando o monóxido de carbono é ligado a hemoglobina, ele assume uma nova conformação irreversível, chamada de carboxihemoglobina. No estado de São Paulo, os cadáveres carbonizados são periciados no Instituto Médico-Legal, mais especificamente no núcleo da Antropologia Forense, área especializada na aplicação do conhecimento antropológico que tem como um dos objetivos identificar o indivíduo e diagnosticar a causa da sua morte. O estudo da concentração de monóxido de carbono no cadáver permite inferir se a carbonização ocorreu ante ou post mortem. Esta informação é altamente importante em termos legais para a aplicação de penalidades, em casos criminais. O objetivo do presente estudo foi caracterizar o perfil epidemiológico das vítimas carbonizadas necropsiadas entre os anos de 2010 e 2015, a fim de estabelecer se o óbito ocorreu antes ou após a carbonização. Os dados de 214 vítimas foram estudados e as informações coletadas foram agrupados de acordo com gênero, faixa etária, raça, local onde o corpo foi encontrado, causa da morte, presença ou ausência do Sinal de Montalti e concentração de carboxihemoglobina. Quanto ao perfil das vítimas, a maioria era do sexo masculino (78,5\%), tinha raça branca $(39,2 \%)$ e idade entre 16 e 47 anos $(90,4 \%)$. A causa da morte variou entre os anos estudados, os corpos foram encontrados com maior frequência nas vias públicas $(36,4 \%)$, em 48,1 \% dos cadáveres o Sinal de Montalti estava ausente e em $33,2 \%$ dos casos a carboxihemoglobina estavam abaixo de $9 \%$. Assim, estes dados permitem concluir que a maioria dos indivíduos estavam sem vida quando ocorreram as lesões pelo fogo, ou seja, a carbonização ocorreu post mortem.
\end{abstract}

DESCRITORES: Antropologia Forense; Carboxihemoglobina; Gases Asfixiantes; Monóxido de Carbono.

\footnotetext{
${ }^{1}$ Biomédica do Programa de Aprimoramento Profissional do Hospital das Clínicas da Faculdade de Medicina da Universidade de São Paulo; letyciaandrade@hotmail.com

2. Médico Legista, Diretor Técnico de Serviço Núcleo de Antropologia Forense do Instituto Médico Legal de São Paulo; tieppoalves@uol.com.br

3. Médico Legista, Núcleo de Antropologia Forense do Instituto Médico Legal do Estado de São Paulo; lrfontes@uol.com.br

4. Bióloga do Laboratório de Investigação Médicas do Hospital das Clínicas da Faculdade de Medicina da Universidade de São Paulo, Doutora em Ciências pela Fac. de Medicina da Universidade de São Paulo; ana.mielli@hc.fm.usp.br

Endereço para correspondência: Dra. Ana Cristina Mielli. Rua Teodoro Sampaio, 115 - Instituto Oscar Freire, FMUSP - São Paulo, SP - 05405-000
} 


\section{INTRODUÇÃO}

$\mathbf{N}$

o processo de investigação forense, a falta

de provas e/ou o estado de deterioração

de um corpo são fatores que podem dificultar a confirmação da causa de morte em cadáveres carbonizados. Sendo assim, é fundamental diagnosticar outros ferimentos além daqueles causados pelo fogo, já que a queima de corpos é uma conhecida estratégia utilizada por criminosos para dissimular os homicídios ${ }^{1}$.

Em toda a história da humanidade, cadáveres carbonizados são normalmente usados para encobrir provas, bem como dificultar a identificação da vítima; no entanto, outras possibilidades devem ser consideradas, como a causa natural de morte seguida de acidentes de incêndio. A determinação da carboxihemoglobina $(\mathrm{COHb})$ associada a outros achados periciais, como a fuligem no trato respiratório, por exemplo, é uma análise importante nesses casos ${ }^{2}$.

O monóxido de carbono ( $\mathrm{CO}$ ), formado pela combustão incompleta de hidrocarbonetos, é um gás altamente perigoso para os seres humanos por ser incolor, inodoro, insípido, não irritante, não inflamável e tóxico. Sendo facilmente absorvido através dos pulmões, o $\mathrm{CO}$ inalado resulta na formação da $\mathrm{COHb}$ devido à alta afinidade do $\mathrm{CO}$ à molécula de hemoglobina $(\mathrm{Hb})$. Este processo desloca o oxigênio $\left(\mathrm{O}_{2}\right)$ da $\mathrm{Hb}$, reduzindo o transporte de $\mathrm{O}_{2}$ para os tecidos e resultando em lesão por hipóxia. A toxicidade se faz dependente de sua concentração ${ }^{3}$.

A queima de qualquer combustível libera fumaça, que é a combinação dos gases com as partículas emitidas. Sua produção depende de dois processos: pirólise, que é a liberação de elementos do combustível, sendo originado exclusivamente pela ação do calor, e oxidação, que é o processo em que o oxigênio reage quimicamente com moléculas do combustível, quebrando-as em compostos menores e resultando na produção de luz e calor ${ }^{4}$. A inalação desses resíduos possibilita o achado pericial de fuligem nas vias respiratórias, visualizada como conteúdo escuro na traqueia e brônquios. Esse fenômeno é chamado Sinal de Montalti ${ }^{4,5}$.

Frequentemente as causas de óbito em vítimas com lesões inalatórias estão relacionadas à intoxicação por CO. A gravidade da intoxicação dependerá da concentração de $\mathrm{CO}$, da ventilação do local onde o gás se encontra, da duração da exposição, da metabolização e da função pulmonar do indivíduo que está em contato com o agente tóxico. Exposições a concentrações muito elevadas podem causar debilidade de membros inferiores impossibilitando, assim, o escape da vítima e, consequentemente, causando morte por intoxicação e asfixia $^{6}$.

$\mathrm{Na}$ literatura científica a forma mais comum de carbonização deve-se a causas acidentais. Pesquisas realizadas em alguns países europeus, como França e Alemanha, mostram que mais de $50 \%$ das perícias médicas realizadas em cadáveres deram a carbonização como acidental, restando uma pequena porcentagem para suicídios e homicídios?.

O Instituto Médico-Legal de São Paulo (IML-SP) recebe cadáveres carbonizados para a necropsia, que é realizada pela equipe do Núcleo de Antropologia Forense. As circunstâncias de morte são bem distintas, tais como: catástrofes aéreas, colisões de automóveis e incêndios, podendo estes serem criminosos e intencionais ou não ${ }^{8-}$ ${ }^{10}$. Porém, não é raro o encontro de corpos que foram incendiados após a morte, na tentativa de se ocultar o cadáver, para mascarar a verdadeira causa da morte e dificultar a identificação do corpo ${ }^{11,12}$

Achados como fuligem nas vias respiratórias e alta concentração de $\mathrm{COHb}$ no sangue da vítima constituemse em importantes elementos que possibilitam afirmar se a vítima estava viva ou morta quando entrou em contato com a ação do fogo, eliminando a possibilidade de simulação e podendo ser um fator determinante para o diagnóstico da causa da morte ${ }^{4,6}$.

\section{OBJETIVO}

Caracterizar o perfil epidemiológico das vítimas carbonizadas necropsiadas pelos médicos legistas do Núcleo de Antropologia Forense do IML-SP entre os anos de 2010 e 2015, a fim de estabelecer se o óbito ocorreu antes ou após a carbonização da vítima.

\section{MATERIAL E MÉTODOS}

O estudo incluiu a análise retrospectiva dos laudos que foram elaborados pela equipe do Núcleo de Antropologia, do Instituto de Médico-Legal (IML) de São Paulo, entre os anos de 2010 e 2015. Foram compilados: idade, gênero, raça, concentração de $\mathrm{COHb}$, presença do Sinal de Montalti, local e causa da morte. Projeto aprovado pela CAPPesq HCFMUSP n ${ }^{\circ} .0114 / 09$ (IML-SP/LIM-40-HCFMUSP).

\section{RESULTADOS}

Entre 2010 e 2015, 214 vítimas carbonizadas foram necropsiadas, sendo $168 \quad(78,5 \%)$ do sexo masculino, $44(20,5 \%)$ do sexo feminino e dois casos de sexo indeterminados (1\%) devido à deterioração dos corpos pelo fogo.

Foi observado predomínio da raça branca (39,2\%), seguido por pardos $(26,5 \%)$ e negros $(7,0 \%)$. Não há casos de amarelos e há um percentual de indeterminados $(27,5 \%)$ que podem estar relacionados com o estado de carbonização das vítimas.

Ao avaliar os resultados de acordo com a faixa 
etária, observa-se no gráfico 1 , que a maioria das vítimas tinha idade entre 32 e 47 anos (60,5\%), seguida de 16 a
31 anos $(29,9 \%)$. A idade média de todos os casos foi de 35,9 anos, variando de zero a 90 anos.

Gráfico 1. Distribuição em percentagem com relação à faixa etária das vítimas carbonizadas necropsiadas entre 2010 e 2015 pelo IML do Estado São Paulo

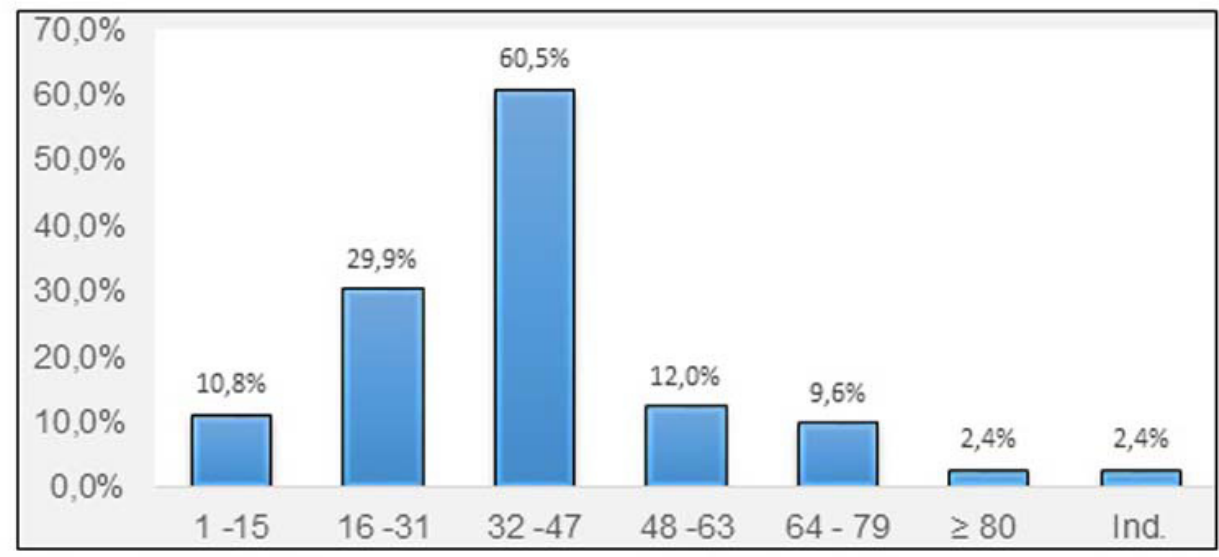

Ind.= indeterminado

No Gráfico 2, observa-se que no ano de 2010 a maioria das causas de morte resultaram da ação de instrumentos perfuro-cortantes e/ou perfurocontundentes. Entretanto, nos anos de 2011, 2012 e 2013 a maioria foi relacionada com intoxicação por $\mathrm{CO}$, que sofreu uma queda nos anos seguintes. Em 2014 a maioria das necropsias apresentaram causa indeterminada da morte e em 2015 a maior parte das vítimas veio a óbito por ação de instrumento perfuro-cortante. Os casos de asfixia presentes no gráfico referem-se a asfixia mecânica diferente da asfixia química provocada pelo $\mathrm{CO}$.
Segundo os laudos, os corpos foram encontrados com maior frequência em vias públicas $(36,4 \%)$, em residências $(32,2 \%)$ e, por fim, em meios de transporte $(22,4 \%)$. Foram descritos também outros locais, menos frequentes e comuns, que apresentaram um percentual de $8,9 \%$ dos casos.

Nos casos analisados o Sinal de Montalti estava ausente em $48,1 \%$ e presente em pouco mais de $30 \%$. Em $19,2 \%$ das vítimas não se pode determinar se havia ou não fuligem nas vias aéreas.

Gráfico 2. Distribuição em percentagem com relação a provável causa de morte das vítimas carbonizadas necropsiadas entre 2010 e 2015 pelo IML de São Paulo.

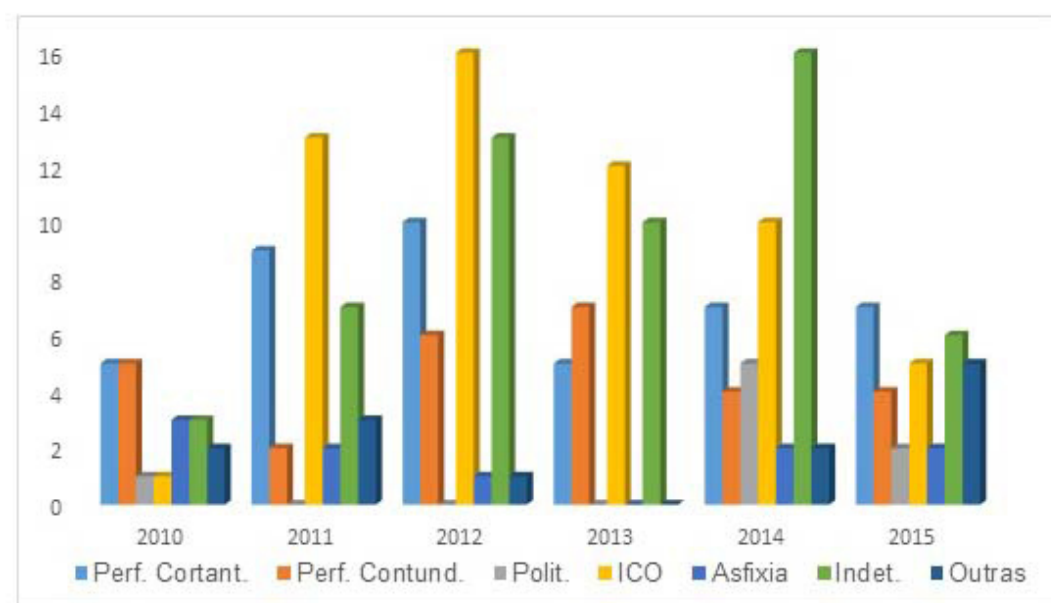

Perf. - Cortant. $=$ Perfuro-cortante; perf. - contund. $=$ perfuro-contundente; polit. $=$ poli traumatismo; $\mathrm{ICO}=$ Intoxicação por $\mathrm{CO} ;$ Asfixia = asfixia mecânica; indet. = indeterminado. 
No Gráfico 3, pode-se observar que a concentração de $\mathrm{CO}$ predomina na faixa de $0-9 \%$ $(33,2 \%)$, posteriormente a faixa de $10-20 \%(12,6 \%)$.
Houve muitos casos sem análise da $\mathrm{COHb}(34,1 \%)$ por amostragem prejudicada para essa análise.

Gráfico 3. Distribuição em percentagem com relação à concentração de $\mathrm{COHb}$ das vítimas carbonizadas necropsiados entre 2010 e 2015 pelo IML de São Paulo

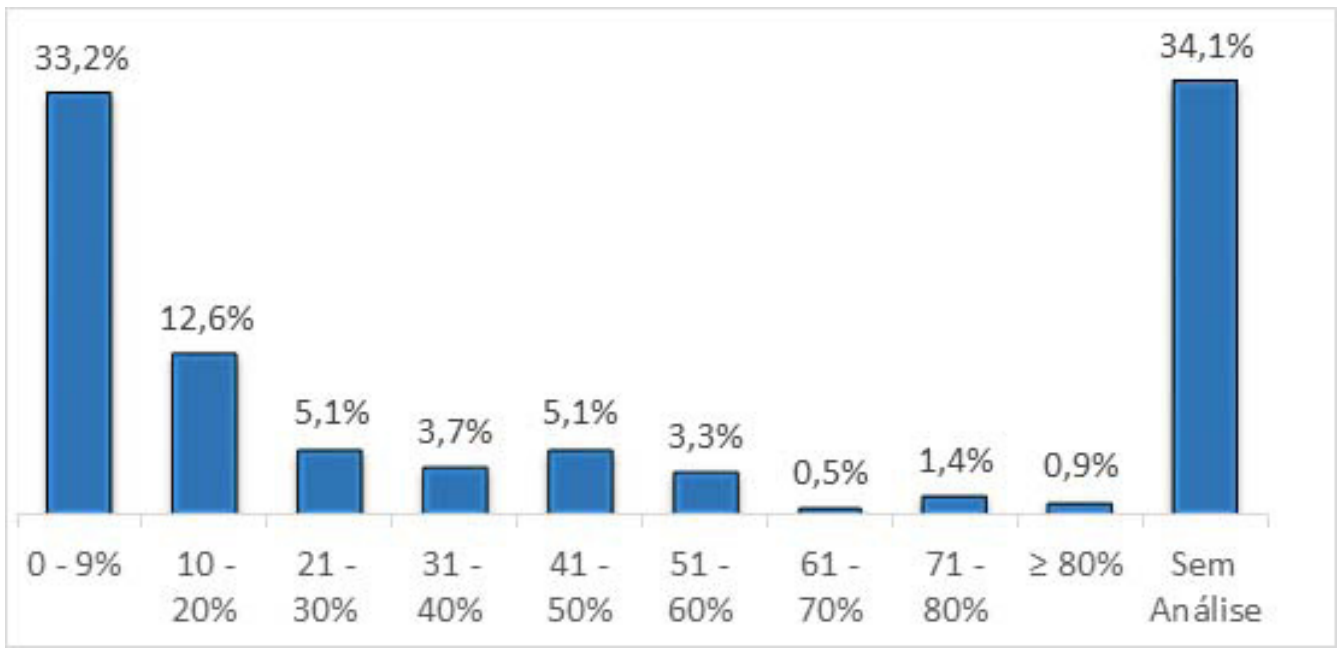

\section{DISCUSSÃO}

Este trabalho foi realizado a partir da leitura direta e compilação dos dados dos laudos das vítimas necropsiados com sinais e lesões por fogo no IML-SP. Os resultados apresentados evidenciaram uma considerável prevalência de vítimas com baixa concentração de $\mathrm{COHb}$, assim como ausência do Sinal de Montalti.

Foram registrados 214 casos e constatado que em $65,9 \%$ das vítimas foi solicitada a realização da análise de $\mathrm{COHb}$. Dentre as vítimas submetidas à dosagem de $\mathrm{COHb}, 33,2 \%$ encontrava-se abaixo de $9 \%$ e em $32,6 \%$ dos casos a concentração de $\mathrm{COHb}$ encontrada estava na faixa de 10 a 80\%. De acordo com Marshall et al. (1995) indivíduos não fumantes expostos ao $\mathrm{CO}$ apresentam concentrações de $\mathrm{COHb}$, sem relato de danos, entre $0,4 \mathrm{e}$ $6,0 \%$ e indivíduos com nicotina e/ou seus metabolitos no sangue variam a concentração de $\mathrm{COHb}$ entre 1 e $7 \%{ }^{13-15}$.

A partir das análises antropológica dos corpos carbonizados, pode-se observar que houve predomínio do sexo masculino $(78,5 \%)$, em relação ao sexo feminino $(21,5 \%)$, assim como houve predomínio da raça branca (39,2\%). Observou-se também, um percentual de casos indeterminados $(27,5 \%)$ em relação à determinação da raça, o que estava relacionado com a dificuldade da visualização desse fator em cadáveres que sofrem ação do fogo. Estudo realizado por Soares (2008) demonstrou que cerca de $90 \%$ dos corpos carbonizados em Ribeirão Preto até o ano de 2006 pertenciam ao sexo masculino e eram brancos ${ }^{16}$, Rodge (1995) verificou que na Noruega $70 \%$ dos casos analisados pertenciam ao gênero masculino ${ }^{15}$.
A prevalência etária das vítimas carbonizadas foi de 16 a 47 anos (90,4\%), sendo considerado uma população jovem. Em estudo realizado em Belo Horizonte (MG), com vítimas carbonizadas, também foi encontrado predomínio entre jovens adultos. Esse fato pode estar ligado à faixa etária da população brasileira, que é considerada majoritariamente jovem, podendo ser correlacionado com acidentes de trânsito, ou mesmo a ligação com o crime, como por exemplo, os homicídios ${ }^{4}$.

$\mathrm{Na}$ investigação forense, a determinação da causa da morte, após a realização da perícia necroscópica e as informações obtidas sobre o local onde o corpo foi encontrado, pode ajudar a solucionar muitos casos. Neste estudo, observou-se que os corpos carbonizados foram encontrados com maior frequência nas vias públicas $(36,4 \%)$, seguido pelas residências $(32,2 \%)$ e por fim, nos meios de transportes $(22,4 \%)$, como automóveis, ônibus e aeronaves. Cadáveres encontrados no interior de veículos podem ter sido carbonizados de forma acidental ou não, porém quando o corpo é encontrado no porta-malas de um veículo ou em locais afastados como matagais, distantes da margem da rodovia ou vias públicas, é via de regra, um caso de homicídio. Entretanto, quando se trata de um corpo carbonizado encontrado em residência, a causa pode ser acidental, homicida ou até mesmo suicida ${ }^{9}$.

É importante o relato de lesões existentes no cadáver pelo médico legista durante o exame necroscópico, possibilitando determinar o momento em que o indivíduo entrou em contato com a ação do fogo $0^{4,14}$. Neste estudo, há vários casos de mortes por consequência de ação de instrumentos perfuro-cortantes e perfuro-contundentes 
(2010 e2015), além de outras causas relatadas anteriormente, assim como causas indeterminada da morte (2014), e esse fato pode estar associado com a dificuldade e complexidade de realizar-se um exame necroscópico em vítimas carbonizadas ${ }^{4,11}$.

Outro fator importante observado durante a necropsia é se há presença do Sinal de Montalti, sendo uma das características apresentadas por indivíduos que inalam a fumaça por um certo período de tempo. Segundo Souza et al. (2004) a lesão inalatória pode aumentar cerca de $20 \%$ a chance de mortalidade quando associada a queimaduras ${ }^{17}$. Neste estudo, observou-se a ausência de Sinal de Montalti em 48,1\% das vítimas sendo que, em pouco mais de $30 \%$ dos casos esse fenômeno estava presente. Foi observado também que em alguns casos a visualização de fuligem nas vias aéreas superiores não foi possível (19,2\%), devido a ação do fogo no corpo da vítima.

Estudos realizados anteriormente relatam que pode ocorrer variações entre a concentração de $\mathrm{COHb}$ e a presença do sinal de Montalti, sendo que em alguns casos a vítima pode apresentar concentração de $\mathrm{COHb}$ elevada, porém não apresenta a fuligem nas vias respiratórias, assim como a concentração de $\mathrm{COHb}$ poderá estar baixa e o indivíduo apresentar fuligem nas vias respiratórias ${ }^{15}$. Valores para carboxihemoglobina acima de 10\% indica para o médico legista que o indivíduo respirou no foco do incêndio, entretanto, a morte das vítimas com queimaduras ocorre não somente pela asfixia por $\mathrm{CO}$, mas pelo tipo de queimadura. Na maioria das vezes as vítimas morrem devido à desidratação (queimaduras de segundo e terceiro graus) ou pela carbonização (queimadura de quarto grau) quando há grande perda de tecidos moles e ósseo, modificando a estrutura e a forma, transformando parcial ou totalmente os tecidos em carvão e cinza grau ${ }^{5}$.
A intoxicação por CO é uma das principais causas de morte por intoxicação no mundo ${ }^{18}$. Em Portugal, durante um estudo foi relatado que 5,86/100.000 habitantes são internados apresentando a sintomatologia causada pela intoxicação ${ }^{19}$. A incidência de casos, acontece com maior intensidade durante o período do inverno, já que as famílias tendem a utilizar mais lareiras, aparelhos de aquecimento para o ambiente e consequentemente, diminuem a ventilação domiciliar ${ }^{20}$. Nos Estados Unidos anualmente acontecem cerca de 1.500 mortes por intoxicação com CO de forma acidental e 2.300 casos por suicídio e 10.000 indivíduos necessitam de cuidados médicos ou perdem pelo menos um dia de trabalho durante o ano pelas complicações causadas com a exposição a esse agente tóxico. Por esse motivo foi criado um programa de combate a exposição ao $\mathrm{CO}$, no qual deve-se instalar detectores de fumaça em locais que o indivíduo permaneça por muito tempo e não apresente ventilação adequada, na tentativa de diminuir o número de casos de intoxicação, sendo ou não fatais ${ }^{21}$.

\section{CONCLUSÃO}

Concluímos neste estudo que as vítimas carbonizadas têm predominância do sexo masculino (78,5\%), prevalência da raça branca $(39,2 \%)$, a maioria das vítimas tinha idade entre 16 e 47 anos (90,5\%), 36,4\% dos corpos foram encontrados em vias públicas $(36,4 \%)$ e a causa da morte variou entre os anos estudados. Em $33,2 \%$ dos casos analisados, foram encontradas baixas concentrações de $\mathrm{COHb}$ (inferiores a 9\%) e ausência do sinal de Montalti (48,1\%), essas características indicam que muitos indivíduos já estavam sem vida no momento que ocorreram as lesões pelo fogo, ou seja, o processo de carbonização ocorreu post mortem.

Andrade LP, Alves PST, Fontes LR, Mielli AC. Characterization of the epidemiological profile of carbonized victims necropsied in IML-SP between 2010 and 2015. Saúde, Ética \& Justiça. 2017;22(1):3-8.

\begin{abstract}
Carbon monoxide is part of the group of gaseous pollutants and can act as a powerful asphyxiant, being responsible for high mortality rates in the world. When inhaled, this substance diffuses through the alveolar-capillary membrane. Once in the bloodstream, it readily binds to hemoglobin, due to carbon monoxide's high affinity to this molecule. Consequently, when carbon monoxide is bound to hemoglobin, it takes on an irreversible conformation, called carboxyhemoglobin. In the State of São Paulo, charred victims are investigated at the Medical-Legal Institute, more specifically, at the division of Forensic Anthropology, an area specialized in the application of anthropological knowledge. This information is highly important in legal terms for the application of penalties in criminal cases. The aim of this study was to characterize the epidemiological profile of charred victims necropsied in the Medical-Legal Institute of São Paulo between 2010 and 2015, in order to establish if the death occurred before or after carbonization. Data from 214 victims were studied and the information collected was grouped according to gender, age group, skin color, place where the body was found, cause of death, presence or absence of the Montalti Signal, and carboxyhemoglobin concentration. The results showed that the majority were male (78.5\%), Caucasians (39.2\%), and aged between 16 and 47 years old $(90.4 \%)$. The cause of death varied among the years, charred bodies were found more frequently in public roads (36.4\%), in $48.1 \%$ of the bodies the Sign of Montalti was absent, and in $33.2 \%$ of the cases the carboxyhemoglobin was below $9 \%$. These data thus allow us to conclude many individuals were already lifeless at the time of fire injuries, that is, the charred occurred post mortem.
\end{abstract}

KEY WORDS: Forensic Anthropology; Carboxyhemoglobin Asphyxiating Gases; Carbon Monoxide. 


\section{AGRADECIMENTOS}

Ao Instituto Médico Legal de São Paulo (IML-SP) e ao Laboratório de Investigação Médica (LIM-40-HCFMUSP).

\section{REFERENCIAS}

1. Popovic VM, Atanasijevic TC, Nikolic SD, Micic JR Concentration of carbon monoxide in carbonized bodiesForensic aspects. Leg Med(Tokyo). 2009;11(Suppl 1):S318 20. DOI: http://dx.doi.org/10.1016/j.legalmed.2009.01.045

2. Tümer AR, Akçan R, Karacaoğlu E, Balseven-Odabaşi A, Keten A, Kanburoğlu C, et al. Postmortem burning of the corpses following homicide. J Forensic Legal Med. 2012;19(4):223-8. DOI: http://dx.doi.org/10.1016/j. jflm.2012.01.001

3. Boechat J L, Rios J L. Poluição de Ambientes Internos. Rev. Bras. Alerg. Imunopatol. 2011;34(3):83-9.

4. Gerostamoulos D, Beyer J, Wong K, Wort C, Drummer $\mathrm{OH}$. Carbon monoxide concentrations in the 2009 Victorian Bushfire disaster victims. Foresinc Sci Int. 2011;205(1-3):69-72. DOI: http://dx.doi.org/10.1016/j. forsciint.2010.08.029

5. Montenegro JB, Leal JLF, Cruz DB, Carvalho MVD, Souza EHA, Vasconcelos BCE. Perícia nas Vítimas de Carbonização. Derecho y Cambio Social. 2013;32:1-11

6. Xavier DM, Dau LIG, Nunes PD, Ramos MG, Roquette ALB, Santos A. Correlação entre cadáveres carbonizados e a dosagem da carboxihemoglobina. Rev Bras Crimin. 2015;4(1):19-25. DOI: http://dx.doi.org/10.15260/rbc. v4i1.79

7. Olson KN, Hillyer MA, Kloss JS, Geiselhart RJ, Apple FS. Accident or arson: is CO-oximetry reliable for carboxyhemoglobin measurement postmortem. Clin Chem. 2010;56(4):515-9. DOI: http://dx.doi.org/10.1373/ clinchem.2009.131334

8. Sociedade Brasileira de Hipertensão. Derrame, infarto e homicídio são as principais causas de mortes no país [Internet]. São Paulo; 2008 [Acesso em 2016 jun. 08]. Disponível em: http://www.sbh.org.br/geral/noticias. asp?id=141

9. Poisson P, Chapenoire S, Schuliar Y, Lamant M, Corvisier JM. Four major disasters in Aquitaine use of odontologic techniques for identification. Am J Forensic Med Pathol. 2003;24(2):160-3

10. Chapenoire S, Schuliar Y, Corvisier JM. Rapid, efficient dental identification of $92 \%$ of 13 train passengers carbonized during a collision with a petrol tanker. Am J Forensic Med Pathol. 1998;19(4):352-5.

11. Jordão NQ, Santiago APACS, Montenegro JB. Identificação de Corpos Carbonizados no IML-PE. Derecho y Cambio Social. 2013;31:1-10.

12. Valenzuela A, Martin-de las Heras S, Marques T, Exposito $\mathrm{N}$, Bohoyo JM. The application of dental methods of identification to human burn victims in a mass disaster. Int J Legal Med. 2000;113(4):236-9.

13. Marshall MD, Kales SN, Christiani DC, Goldman RH. Are reference intervals for carboxyhemoglobin appropriate? A survey of Boston area laboratories. Clin Chem. 1995;41(10):1434-8.

14. Siqueira MEPB, Martins I, Costa AC, Andrade EL, Esteves MTC, Lima SA. Valores de Referências para Carboxiemoblogina. Rev Saúde Pública. 1997;31(6):618-23. DOI: http://dx.doi.org/10.1590/S003489101997000700010

15. Rodge S, Olving JH. Characteristics of fire victims in different sorts of fires. Forensic Sci Intern.1996;77(1996):93-9.

16. Soares ATC, Guimarães MA. Dois anos de Antropologia Forense no centro de Medicina Legal (CEMEL) da Faculdade de Medicina de Ribeirão Preto-USP. Medicina (Ribeirão Preto). 2008;41(1):7-11. DOI: http://dx.doi. org/10.11606/issn.2176-7262.v41i1p7-11

17. Souza R, Jardim C, Salge JM, Carvalho CRR. Lesão por inalaçãodefumaça.JBrasPneumol.2004;30(6):557-65.DOI: http://dx.doi.org/10.1590/S1806-37132004000600011

18. Cardiga R, Proença M, Carvalho C, Costa L, Botella A, Marques $\mathrm{F}$, et al. Intoxicação por monóxido de carbono com compromisso cardíaco: o que sabemos? Rev Port Cardiol. 2015;34(9):557(e1-557.e5). DOI: http://dx.doi. org/10.1016/j.repc.2015.01.006

19. Sá MC, Rodrigues RP, Moura R, Internamentos por intoxicação com monóxido de carbono em Portugal. Acta Med Port. 2011;24(5):727-34.

20. Ruas F, Mendonça MC, Real FC, Vieira DN, Teixeira HM. Carbon monoxide poisoning as a cause of death and differential diagnosis in the forensic practice: A retrospective study, 2000-2010. J Forensic Leg Med. 2014;24:1-6. DOI: http://dx.doi.org/10.1016/j.jflm.2014.02.002

21. Homer CD, Engelhart DA, Lavins ES, Jenkins AJ. Carbon Monoxide - related deaths in a metropolitan county in the USA: an 11-years study. Forensic Sci Int. 2005;149(2-3):159-65. DOI: http://dx.doi.org/10.1016/j. forsciint.2004.06.012 